\title{
Intelligente Prozeßautomatisierung durch moderne Sensorik, graphische Programmierung und die Nutzung von Netzwerkdiensten
}

\author{
Prof. Dr.-Ing. habil. Volkmar Kirbach, Dipl.-Ing. Jens Volker Steinert
}

Summary: The combination of the graphic based developping system LabView with field bus elements allows to find high effective solutions in process and laboratory automation. For one kind of field bus elements, the ADAM-modules from Advantech, the connection to LabView was realized in the Windows 95 environment. Further researches and applications will allow remote monitoring and remote control via intranet and internet.

\section{Aktueller Stand und Entwicklungstrends auf dem Gebiet der Prozeßautomatisierung}

Im Umfeld der Prozeßautomatisierungssysteme fanden in den letzten Jahren umwälzende Änderungen statt. Die klassische Automatisierungstechnik befindet sich im Umbruch. Die etablierten Systeme sind an der Kostenund Leistungsgrenze angelangt. Zusätzliche Anforderungen verlangen nach neuen Konzepten.

Dazu gehören mehr Flexibilität, sowie die Möglichkeit, mit Standardwerkzeugen nach Kundenwunsch Anpassungen an Steuerungen durchzufuihren.

Mehr und mehr Automatisierungsfunktionen werden durch Lösungen außerhalb des Automatisierungssystems wahrgenommen. Das Ziel einer solchen Entwicklung ist es, Automatisierungsstrukturen modular und damit kostenguinstig und kundenorientiert aufzubauen. Diese Automatisierungsstruktur muß einen „SystemKern" und offengelegte, standardisierte Schnittstellen für die spätere Erweiterung mit zusätzlichen Funktionen enthalten. Der modulare Aufbau ermöglicht maßgeschneiderte Automatisierungslösungen.

Mit Hilfe der Feldbustechnologie wird versucht, die Kosten auf der Ein-/Ausgabeebene für Prozeßdaten (Prozeßperipherie) zu reduzieren. Derzeit machen sie noch etwa 1/3 der Automatisierungskosten aus. Weniger komplexe Automatisierungsfunktionen, wie beispielsweise Meßdatenvorverarbeitung und einfache Regelungen können damit in die Feld-(Prozeß-)Ebene verlagert werden.

Für die Zukunft kristallisieren sich Automatisierungslösungen mit folgenden Eigenschaften heraus:

- dezentrale Struktur

- modularer Aufbau, leicht erweiterbar

- graphische Oberfläche / graphische Programmierung

- PC-Technik (Plug \& Play) als Prozeßrechner

- Netwerkfähigkeit mit Internet-Zugang

- Betriebssystem Windows NT

- Echtzeitdatenbanken mit verteilter Datenbankstruktur

\section{Möglichkeiten der Meßdatenerfassung mit intelligenten Sensormodulen unter LabView}

In der industriellen Praxis und in Labors steht oft die Aufgabe, Meßdaten unterschiedlichster Herkunft zu erfassen und zu verarbeiten.

Diese Meßdaten fallen örtlich verteilt und nach den verschiedensten Meßprinzipien bei unterschiedlichen Signalpegeln an (z.B. Ströme, Spannungen, Impulse). Ein Prämisse für Überwachungs-, Prozeßsteuerungs- und -leitaufgaben ist es, daß die Meßdaten in digitalisierter Form einem Prozeßrechner zur Weiterverarbeitung zur Verfuigung gestellt werden.

Um ein integriertes Prozeßdatenerfassungs- und -verarbeitungssystem zu installieren und dabei mit möglichst wenig Aufwand auszukommen, hat es sich als vorteilhaft erwiesen, die Meßdaten über ein lokales Feldbussystem zu sammeln und weiterzuleiten.

Dabei werden die zum Einsatz kommenden Sensormoduln über eine abgeschirmte Zweidrahtleitung (RS 485-Bus) miteinander verbunden und mit einem Steuerrechner gekoppelt. Die Sensormoduln sind in der Lage, mehrere Signale der unterschiedlichsten Meßdatengeber aufzunehmen, umzuwandeln und weiterzuleiten. Die Meßwerte werden auf Anfrage als Zeichenketten über die Busleitung zum Prozeßrechner übertragen. Das hat außer der Ersparnis von Kabel- und Verlegekosten den Vorteil, daß über die Datenleitung keine durch Störeinflüsse verursachten Meßfehler übertragen werden können. Im ungünstigsten Fall muß die Übertragung einzelner Zeichenketten wiederholt werden oder es kommt ein anderes Übertragungsprotokoll (z. B. MODBus) zum Einsatz.

Ein im Produktionsprozeß bzw. in der Fertigungsanlage verlegtes Feldbussegment darf maximal $1,2 \mathrm{~km}$ lang sein und bedient max. 32 Busteilnehmer (z. B. Meßgeräte mit RS 485-Anschluß oder entsprechende Sensormoduln). Über Repeater kann die Anzahl der Busteilnehmer auf maximal 127 pro physikalischem Busstrang erhöht werden.

Die Übertragungsgeschwindigkeit ist abhängig von der Länge der Übertragungsstrecke. Bei maximaler Länge des Bussegmentes liegt die Übertragungsgeschwindigkeit noch bei $>100 \mathrm{KBit} / \mathrm{s}$.

Durch den gezielten Einsatz von Repeatern lassen sich verschiedene Bustopologien aufbauen. Auf diese Weise sind einfache Linienstrukturen möglich, aber auch Abzweigungen (Stern-Topologie). Um Signalreflektionen auf dem Bus zu vermeiden, ist jedes Bussegment am Anfang und Ende mit einem Wellenwiderstand abzuschließen. Dieser Abschlußwiderstand wird zwischen 
die beiden Busleitungen (A und B) geschaltet. Zusätzlich wird die Busader A über einen sog. Pull-Up-Widerstand an Potential und die Busader B über einen Pull-DownWiderstand auf Masse (Data-Ground) gelegt. Diese Widerstände sorgen fuir ein definiertes Ruhepotential bei fehlender Datenübertragung auf dem Bus.

Die Kommunikation zwischen dem Bus und dem Prozeßrechner (oft PC) erfolgt physikalisch über einen RS485/RS232-Konverter oder uiber eine RS485-Schnittstelle. Logisch kommt ein bestimmtes Protokoll zum Einsatz, mit dem die Busteilnehmer mit dem Rechner bzw. untereinander kommunizieren.

Im Labor für Prozeß- und Anlagenautomatisierung der TFH Wildau werden bisher Sensormoduln verwendet, die das ASCII- und MOD-Bus-Protokoll unterstuitzen. Speziell das relativ transparente ASCII-Protokoll läßt sich am einfachsten in einer Programmiersprache umsetzen[1].

Es stehen einerseits Sensormodule für den variablen Einsatz und unterschiedliche Busprotokolle zur Verfügung (z.B. die ISM-Serie der Fa. Meilhaus), die den Anschluß mehrerer der gängigen Sensortypen erlauben sowie andererseits Sensormodule, die mit Einschränkungen nach dem jeweiligen Anwendungsfall zu konfigurieren sind (Geräte der Fa. Advantech).

Um die Meßdaten zu visualisieren, zu speichern und weiterzuverarbeiten, ist auf dem angeschlossenen Prozeßrechner eine entsprechende Echtzeit-Softwareumgebung zu installieren (oft als Visualisierungssystem bezeichnet). Die Anbieter von Sensormoduln stellen diese in der Regel auch für ihre Gerätetechnik zur Verfügung. Oft funktioniert eine solche Firmware allerdings nur für die Sensormoduln des Herstellers.

Dieser Zustand macht beim Anwender in bestimmtem Umfang eine Software-Eigenentwicklung zur Geräteanpassung, -konfiguration und -steuerung (Driver) in der Feldbusebene erforderlich (Abb. 1).
Um den Entwicklungsaufwand möglichst gering zu halten und eine einheitliche und leistungsstarke Softwareumgebung zu nutzen, wurde das Entwicklungssystem LabView der Fa. National Instruments ausgewählt. Es basiert auf der Methode der graphischen Programmierung und bietet den Vorteil, mit wenig Aufwand bei der Entwicklung von Bedieneroberflächen schnell eine nutzbare Problemlösung verfügbar zu haben|2]. Die Abfrage der Rechner-Schnittstellen stellt den Anwender ebenfalls nicht vor größere Probleme. Außerdem hat man bei Nutzung von LabView über Multi-l/O-Karten direkten Zugriff auf ausgewählte analoge und digitale Meßwerte[7].

Über Internet und Intranet sind mit Hilfe des Entwikklungssystems Labview die perspektivisch wichtigen Automatisierungsaufgaben Fernüberwachung und Fernsteuerung (Remote Monitoring, Remote Control) lösbar.

\section{Umsetzung des Entwicklungskonzeptes mit LabView}

Ziel der Softwareentwicklung an der TFH Wildau war es, auf der Plattform von LabView ein einheitliches, modular aufgebautes und gem. zukünftiger Anforderungen jederzeit erweiterbares Labordatenerfassungs- und -verarbeitungssystem aufzubauen.

An das Entwicklungsergebnis, d.h. die neuen LabViewRoutinen, wurden dabei die folgenden Anforderungen gestellt:

- modularer Aufbau

- unkompliziert erweiterbar und änderbar

- für unterschiedliche Busstrukturen und -protokolle anwendbar

- Einsatz für ein breites Spektrum von Meßaufgaben

- Anwendung für Busstrukturen und DAQ-Systeme

- Vorbereitung der Datenbereitstellung für ein Rechnernetzwerk

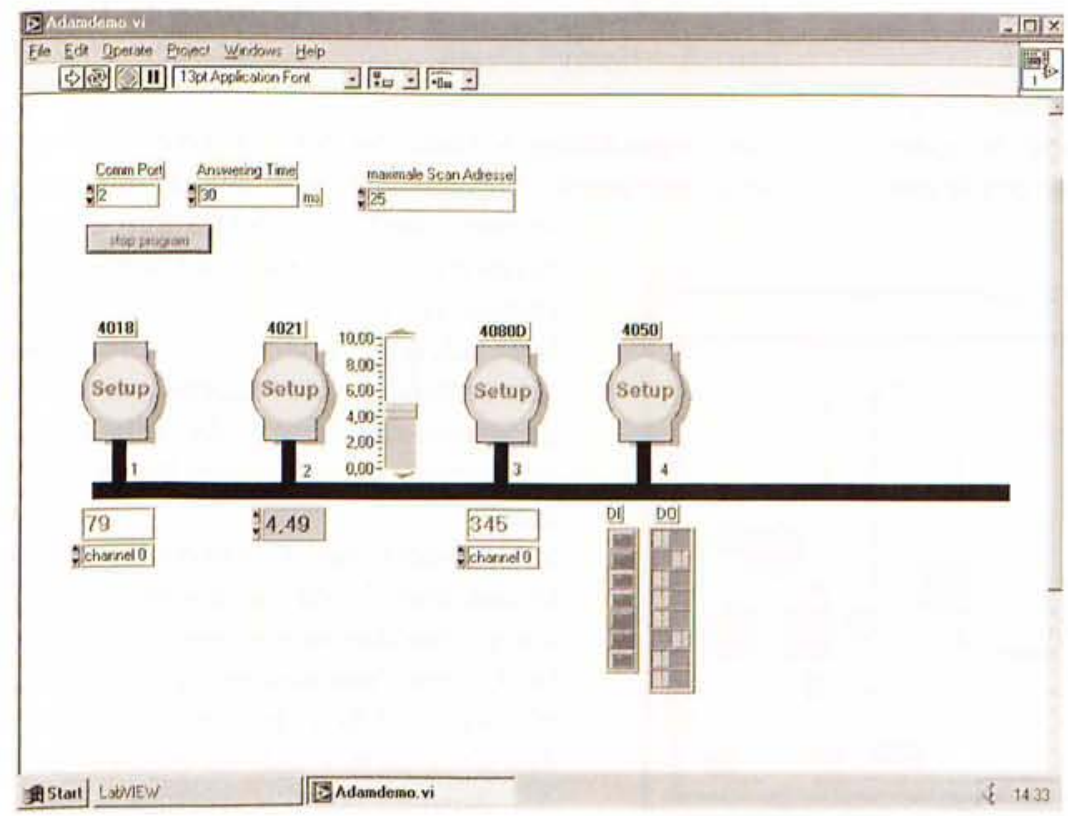

Abbildung 1 : LabView-Darstellung eines modellierten Feldbus-Segments
Das Kommunikationsprotokoll ist für alle im Einsatz befindlichen Sensormodule ausfuihrlich dokumentiert, so daß die Entwicklung der entsprechenden VI's (Virtuelle Instrumente, d. h. Bedienprogramme) zur Verbindungsaufnahme mit den Modulen problemlos möglich ist.

Es wurden für die am häufigsten eingesetzten ADAM-Module der 40-er Serie $\mathrm{VI}$ 's erstellt, mit deren Hilfe sich alle Funktionen der intelligenten Sensormodule ausfuihren bzw. abfragen lassen (Konfiguration des Sensormoduls und der angeschlossenen Ein- und Ausgangskanäle, Abfrage von Meßwerten und Statusgrößen, Setzen von Ausgangssignalen, Überprüfen von Grenzwerten und Erfassung von Alarmen, Kalibrierung der Module u. a.).

Abb. 2 zeigt exemplarisch die mit Hilfe von LabView durchgeführte Konfiguration eines ausgewählten ADAM-Moduls. 


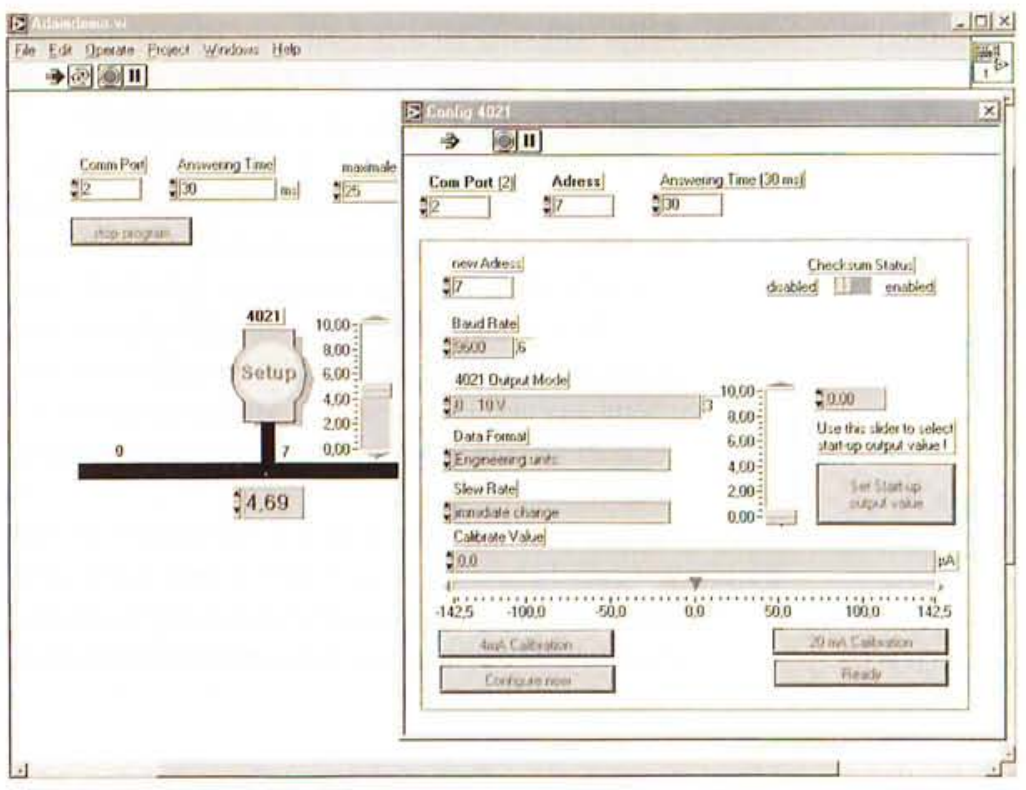

Abbildung 2: Beispiel der Konfigurierung eines ADAM -Moduls

Durch konsequente Anwendung von LabView sowie die Benutzung der neuen VI's reduziert sich der Aufwand für die Entwicklung und den praktischem Einsatz in der Prozeß- und Laborautomatisierung, d. h. für die Aufgaben der Prozeß- und Laborüberwachung, Steuerung und Regelung, um ein Vielfaches.

\section{Lösung eines Anwenderproblems mit Hilfe eines LabView-Programms}

Als Beispiel soll an dieser Stelle die Realisierung einer Füllstandsregelung vorgestellt werden. Die Ein- und Ausgabegrößen des Regelungsvorganges sind der Füllstand und die Drehzahl der Förderpumpe als Stellgröße.

Das gesamte LabView-Programm zur Lösung dieser Regelungsaufgabe (das Ergebnis der graphischen Programmierung mit Hilfe der Sprache G) ist in Abb. $3 \mathrm{zu}$ sehen.

Es wird deutlich sichtbar, daß der Aufwand sich im wesentlichen auf das Plazieren der Ein- und Ausgabebausteine, der Skalierungsfunktionen sowie des Regler-

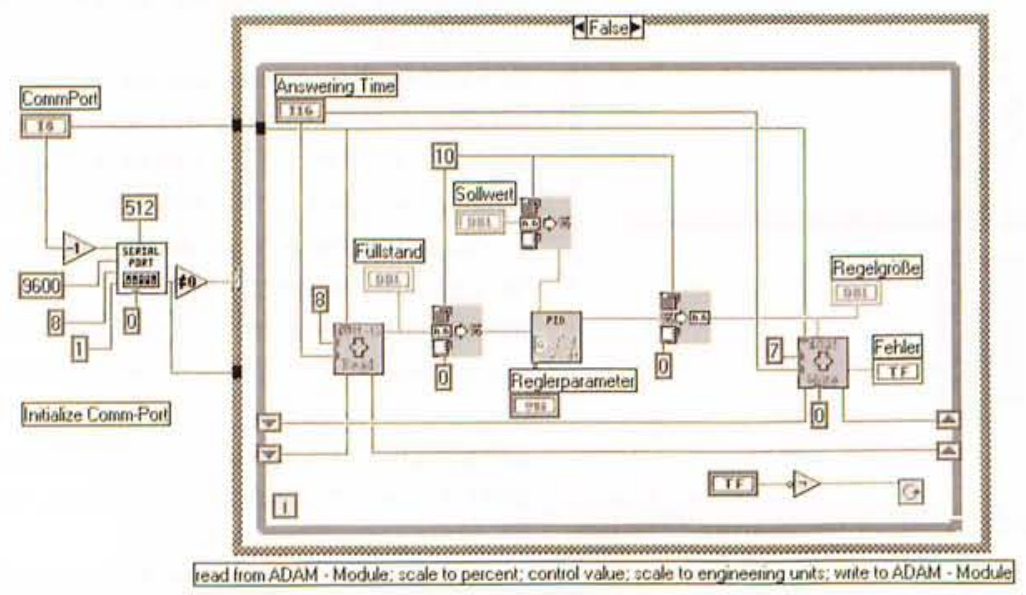

Abbildung 3 : Programm zur Realisierung einer Füllstandsregelung bausteins beschränkt. Werden diese in der geeigneten Weise miteinander verbunden, ist das Programm lauffähig. Im linken Teil der Abbildung ist die Initialisierung der Schnittstelle zu sehen, welche auch in einem separaten Baustein integriert werden kann.

Der Nutzer kommt mit der in der Programmiersprache G notierten Darstellung (eigentliches Programm) nicht in Berührung, er arbeitet mit dem in Abb. 4 dargestellten zugehörigen Bedienpaneel und kann sich somit voll auf die regelungstechnische Problemstellung (in diesem Beispiel die Reglerparametrierung und die Sollwerteinstellung) konzentrieren [5] [6].

Das in LabView konsequent durchgesetzte VISA-Konzept (Virtual Instrumentation Software Architecture) [7] verkörpert einen Meilenstein in der Standardisierung von I/ O-Treibersoftware, da es erstmalig die einfache Interoperabilität einzelner Systemkomponenten verschiedener Hersteller erlaubt.

VISA stellt ein einheitliches, herstellerübergreifendes Fundament für die Entwicklung und Integration von Software-Komponenten wie Gerätetreibern, BildschirmFrontpaneels und Anwendersoftware dar, das unabhängig ist vom Typ des Automatisierungsgerätes und unabhängig von Bussystemen, Betriebssystemen, Programmiersprachen und Netzwerkmechanismen.

Letztlich bedeutet „Virtuelle Instrumentierung “ wesentlich mehr als nur graphische Benutzeroberflächen. Es ist eine Philosophie, mit welcher der Durchbruch im Hinblick auf Produktivität und Effizienz beim Entwurf und praktischen Betrieb von Automatisierungsanlagen erzielt werden kann.

\section{Werkzeuge und Methoden für die Meß- datenerfassung und -verarbeitung in Kom- munikationsnetzen}

Zum gegenwärtigen Stand der Technik gehört die unternehmensweite Nutzung verteilter, offener (oft heterogener) Rechnerarchitekturen in vernetzten Informations- und Kommunikationssystemen.

Dies schließt einfache und komplexe Anwendungen der Prozeß- und Fertigungsautomatisierung ïber öffentliche und private (betriebliche) Datennetze ein.

Schlagworte wie „Connectivity“, „Datenautobahn“ und „weltweite Vernetzung" charakterisieren diesen Trend. In diesem Zusammenhang gewinnen PC-Netze auf Basis Ethernet unter dem Betriebssystem Windows 95 speziell in der Automatisierungstechnik immer mehr an Bedeutung.

Obwohl Ethernet und das CSMA/CDProtokoll (IEEE-Norm 802.3) bzw. mit 


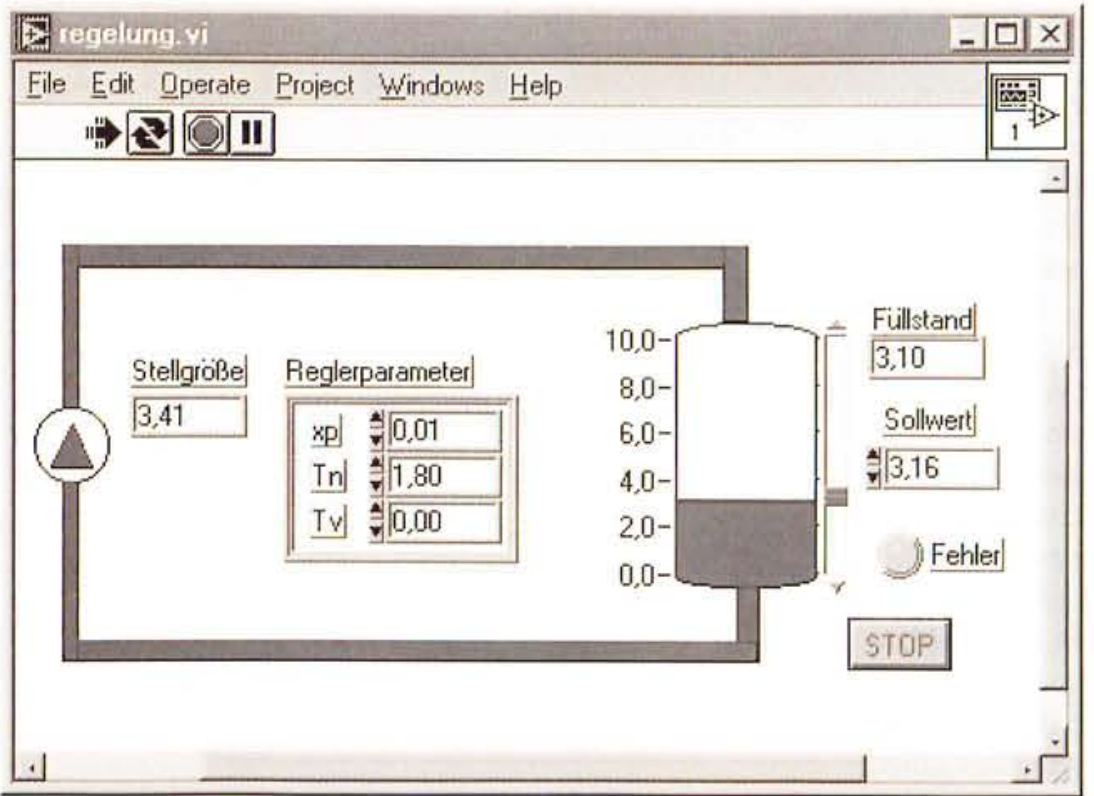

Abbildung 4: Bedienpaneel Füllstandsregelung

TCP/IP-Protokoll als weltweit anerkannter Standard für den unternehmensweiten Datenaustausch in der Vergangenheit hauptsächlich in Büroumgebungen eingesetzt wurden, können die unter Kostendruck stehenden Anwender der Prozeß- und Fertigungsautomatisierung die Vorteile und die Leistungsfähigkeit von Ethernet nicht mehr länger ignorieren.

Das TCP/IP-Protokoll ist als Ethernet-Protokoll am besten für den globalen Datenaustausch geeignet. Dieses Protokoll ist auf alle Rechnerplattformen portierbar und wird für das Internet zur Übertragung von Daten genutzt.

Versionen mit $10 \mathrm{MBit} / \mathrm{s}$ und $100 \mathrm{MBit} / \mathrm{s}$ verfügen über die notwendige Bandbreite auch für komplexe Systeme. Sie liegt etwa um den Faktor 1000 höher als bei seriellen Systemen. Und eine hohe Bandbreite ermöglicht die für die Automatisierungstechnik erforderlichen kurzen Antwort- und Reaktionszeiten.

Einer der großen Vorteile eines solchen Netzwerks ist, daß Prozeß-Meßdaten in „Quasi-Echtzeit“ verfügbar sind.

Erfaßte Daten können gleichzeitig von verschiedenen Anwendungen überwacht und verarbeitet werden. Um Prozeßdaten im Netzwerk verfügbar zu machen, kann man beispielsweise eine vorhandene SPS mit einem Netzwerkinterface und entsprechender Software ausrïsten. Auch wenn ein Industrie-PC zum Einsatz kommt, kann dieser mit einer Netzwerkkarte aufgerüstet und im Ethernet unter TCP/IP betrieben werden.

Eine kostengünstige Alternative bietet die Fa. Intelligent Instrumentation mit einem neuen Ethernet Data Acquisition System (EDAS) an. In einem für rauhe Betriebsbedingungen besonders geeigneten kompakten und robusten Gehäuse stellt dieses System analoge und digitale Schnittstellen (Prozeßperipherie) mit einem direkten Anschluß an das Ethernet zur Verfügung. Der Anschluß an das Netzwerk erfolgt über das kostengünstige 10BaseT (Unshielded Twisted Pair, UTP) Kabel. Für die Kommunikation wird das vom physikalischen Übertragungsmedium bzw. Netzwerk unabhängige Datenü- bertragungsverfahren TCP/IP eingesetzt, bei dem die Daten von Rechner zu Rechner weitergereicht werden, bis der Zielrechner erreicht ist.

Im EDAS integriert ist ein eigener Prozessor mit BIOS und RAM, der die Vorverarbeitung der Daten übernimmt[3].

EDAS arbeitet im Netzwerk als Datenerfassungs-Server. Die angeschlossenen Clients können gleichzeitig auf alle Ein- und Ausgabefunktionen des Gerätes zugreifen. Dabei läßt sich das System sowohl im synchronen als auch im asynchronen Betrieb betreiben. Im synchronen Modus liefert EDAS auf Anfrage die gewünschte Information oder bearbeitet eine bestimmte Task.

Mit der Voraussetzung eines installierten Ethernets bietet das graphische Entwicklungssystem LabView mit der Internet-Erweiterung die Möglichkeit, globale Meßdaten im Netzwerk zur Verfuigung zu stellen (Abb. 5). Mit der Internet-Funktionalität kann man auf die in Windows 95 neu implementierten OLE-2.0-Funktionen (Object Linking and Embedding) zugreifen.

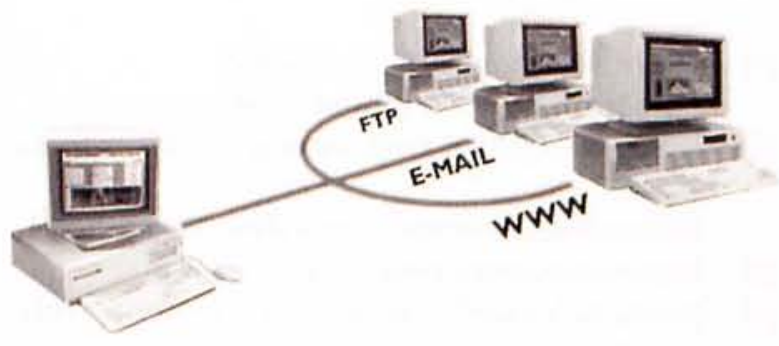

Abbildung 5 : Erweiterte Möglichkeiten von LabView

Insbesondere wird mit der OLE-Automation, einer Erweiterung von DDE (Dynamic Data Exchange), ein Mechanismus nicht nur für den dynamischen Daten- und Befehlsaustausch zwischen verschiedenen Applikationen, sondern auch zwischen den Systemen auf einem Netzwerk bereitgestellt. Weiterhin existiert für LabView ein Zusatzwerkzeug, das folgende neue Web-ServerMöglichkeiten zur Verfiigung stellt [4]:

- Visualisierung von virtuellen Instrumenten (VI ss) über einen Web-Browser

- Sicherheitsmechanismen für die Darstellung von „sensitiven“ Daten

- Erstellung von CGI-Programmen (Common Gateway Interface) in LabView, um Server-Operationen zu realisieren

Senden von elektronischer Post (E-Mail) von einer Applikation aus

- Übertragung von Daten zu einem FTP-Server

Das von der Fa. National Instruments auf der Grundlage von LabView entwickelte SCADA-System BridgeView stellt dem Anwender aus der Automatisierungstechnik 
als Visualisierungs- und Kompaktleitsystem die o.g. Systemkomponenten zur Verfügung.

Mit BridgeView ist es möglich, Meßdaten und Bedienpaneele aus der Produktions- und Fertigungsebene (wie in Abb. 1, 2, 4 dargestellt) im unternehmensweiten Intranet, oder wenn gewünscht, auch weltweit im Internet oder World Wide Web (WWW) zum Zweck der Überwachung und Steuerung bereitzustellen.

Mit Hilfe dieser zukunftsweisenden Technologien lassen sich Anwendungen realisieren, die in der Vergangenheit kaum oder nur unter sehr hohem Aufwand lösbar waren. Insbesondere kann der Benutzer ohne besondere Kenntnisse der zugrundeliegenden Netzwerkstruktur auf beliebige Daten an beliebigen Orten zugreifen.

Klassische Anwendungsgebiete stellen z.B. die nichtlokale Datenüberwachung eines in einem Labor ablaufenden Versuches, die Überwachung eines zentralen Prüfstandes, einer Immobilie im Rahmen des GebäudeManagements oder einer Steuerungsanlage über einen Internet-Browser dar.

Derartige anspruchsvolle Automatisierungsaufgaben sind Gegenstand gegenwärtiger und künftiger Tätigkeit in Forschung und Lehre sowie weiterfuihrender Projekte im Labor Prozeß- und Fertigungsautomatisierung des Fachbereiches Ingenieurwesen/Wirtschaftsingenieurwesen der TFH Wildau.

\section{Literaturangaben}

[1] ADAM 4000 Series User's Manual Advantech Co. Ltd., Taiwan 1994

[2] LabView Graphical Programming for Instrumentation National Instruments Corporation, Austin 1994

[3] http://www.instrument.com/germany

[4] Jamal, R.: Virtuelle Instrumente - gestern, heute, morgen Elektronik, Franzis-Verlag, 1996, Heft 26

[5] Wells, L.; Travis, J.: Das LabView-Buch Prentice-Hall, 1996

[6] Jamal, R.; Erhard, W.: Meßdatenerfassung mit LabWindows Franzis-Verlag, 1994

[7] Jaschinski, H.: VISA - Die Software-Architektur für virtuelle Meßtechnik

Elektronik Industrie 5/96, S. 70-74

\section{Verfasser}

Prof. Dr.-Ing. habil. Volkmar Kirbach Technische Fachhochschule Wildau Fachbereich Ingenieurwesen/Wirtschaftsingenieurwesen Labor Prozeß- und Anlagenautomatisierung

Tel. + 49 (0) 3375/507-130

Dipl.-Ing. Jens Volker Steinert

Technische Fachhochschule Wildau

Fachbereich Ingenieurwesen/Wirtschaftsingenieurwesen

Labor Prozeß- und Anlagenautomatisierung

Tel. + 49 (0) 3375/507-175 